\title{
The Effects of Finger-Walking in Place (FWIP) on Spatial Knowledge Acquisition in Virtual Environments
}

\author{
Ji-Sun Kim \\ Denis Gračanin \\ Center for Human Computer Interaction \\ Department of Computer Science \\ Virginia Tech \\ 2202 Kraft Dr. \\ Blacksburg, VA 24060 \\ \{hideaway, gracanin, quek\}@vt.edu
}

Francis Quek

\begin{abstract}
Spatial knowledge, necessary for efficient navigation, comprises route knowledge (memory of landmarks along a route) and survey knowledge (overall representation like a map). Virtual environments (VEs) have been suggested as a power tool for understanding some issues associated with human navigation, such as spatial knowledge acquisition. The Finger-Walking-in-Place (FWIP) interaction technique is a locomotion technique for navigation tasks in immersive virtual environments (IVEs). The FWIP was designed to map a human's embodied ability overlearned by natural walking for navigation, to finger-based interaction technique. Its implementation on Lemur and iPhone/iPod Touch devices was evaluated in our previous studies. In this paper, we present a comparative study of the joystick's flying technique versus the FWIP. Our experiment results show that the FWIP results in better performance than the joystick's flying for route knowledge acquisition in our maze navigation tasks.
\end{abstract}

\section{Author Keywords}

Virtual environments, spatial knowledge, navigation, locomotion technique

\section{INTRODUCTION}

People use spatial knowledge of the environment for various purposes, such as to find destinations, to give navigation instructions to visitors, to interpret maps, to plan efficient trips, and so forth. These kinds of knowledge can be used to coordinate people's behavior not only in the environment as perceived but also in the environment as cognized (e.g. interpreted and remembered). According to Ittleson et al. [Ittelson1973], the environment is larger than human body and surrounds it. Because people cannot grasp spatial information (e.g., spatial relations or features) of the environment from a single viewpoint, they must explore the environment to integrate spatial knowledge acquired from separate viewpoints and navigating experiences.

Over the last decade, virtual environments (VEs) have been increasingly regarded as an effective medium for studies of human spatial knowledge (or memory) [Stankiewicz2008].
Researchers can provide a convenient, safe and relatively inexpensive method for representing space [Tlauka2008]. However, it has been reported that there are differences in spatial knowledge acquired from a simulated space and a comparable real environment [Chance1998]. These differences are related to virtual locomotion that may induce lower performance in spatial knowledge acquisition relative to walking through an equivalent real environment [Chance1998, Tlauka2008].

Virtual locomotion is usually realized with a keyboard, for desktop VEs, or a joystick (of a wand), for immersive virtual environments (IVEs). We assume that interaction techniques with these types of devices are not directly coupled with navigation tasks as much as walking in the real world. In other words, people may better learn the routes and the layout of the environment when they use embodied abilities that would be overlearned for navigation in the real world.

While it is generally assumed that adopting physical locomotion techniques (e.g., walking-in-place or real walking) leads to higher navigation performance [Bowman2004, Chance1998] (in terms of spatial knowledge acquisition), the full body-based support for these techniques presents many challenges, such as physical body fatigue, large space, expensive simulators, or bodyworn equipments. These physical locomotion techniques are inconvenient and expensive relative to the handoperated techniques using, for example, a keyboard or a joystick.

We considered the trade-offs (e.g., spatial learning performance vs. convenience of user interfaces) of the two types of navigation techniques, and proposed a finger-based locomotion technique. In our previous work, we introduced the Finger-Walking-in-Place (FWIP), for locomotion in a virtual space [Kim2008].

The FWIP is designed by the general observation that people can easily mimic the walking motion by using their fingers because the walking motion of the legs is natural to them. Since humans' spatial knowledge is naturally 
acquired while walking on two legs in a real world, we assume that we may expect some learning effect with the FWIP in a virtual world. The implementation of the FWIP on a Lemur ${ }^{1}$ [JazzMutant] and an iPhone/iPod Touch was evaluated in our previous pilot studies [Kim2008, Kim2009] that showed the multi-touch based FWIP can be used as a reliable locomotion technique in an IVE (i.e., CAVE). In this paper, we present a comparative study of the FWIP technique using a Lemur and iPhone/iPod Touch, versus the joystick's flying technique to investigate the learning effect on spatial knowledge acquisition in a CAVE.

\section{RELATED WORK}

Wayfinding is the cognitive process of problem-solving to find a route or alternative routes for navigation tasks, such as exploration, search, or maneuvering [Bowman2004]. One model related to wayfinding, known as LandmarkRoute-Survey (LRS) model, describes the process of how spatial knowledge is acquired and represented [Siegel1975]. According to the model, people first learn to recognize landmarks in an unfamiliar environment. After they get familiar with these landmarks, they try to learn the procedure to get the route knowledge from one location to the other.

Such route knowledge can be acquired from learning what actions they take as well as remembering landmarks [Cornell1999]. Finally, they can form survey knowledge that is a mental presentation of the environment. Thus it is provided by their sufficient experience in the environment. Even though spatial capability is diverse in individuals, they generally follow the process.

Although navigation is rarely the main task in VEs, effective navigation is necessary to successfully use such environments, including information spaces [Camarata2002]. Thus, success of virtual environments relies heavily on success of locomotion techniques. Since 3D IVEs can provide broad support for human operator's spatial capability, many wayfinding aids have been designed in VEs using different approaches, using wide field of view, physical motion cues, multisensory output, great sense of presence and search methods [Bourdot2002, Bowman2004].

Interaction techniques for navigation tasks have been explored and developed to support these approaches. Bowmann et al. [Bowman2004] classify them in four categories: natural travel metaphors, steering metaphors, target-based metaphors, and manipulation metaphors.

The methods from the first group use physical locomotion. A user can, for example, walk in place [Slater1995, Templeman1999] or on a treadmill [Darken1997,

\footnotetext{
${ }^{1}$ In July 2007, the company has adopted the name of Stantum (http://www.stantum.com), while JazzMutant remains the brand name for Lemur and other products under JazzMutant.
}

Iwat1999], ride a bicycle, or walk in a large sphere [Virtusphere2008]. LaViolla et al. [LaViola2001] propose a hand-free navigation technique which combines a leaning technique for small and medium scale movements and foot gestures to control large scale movements. However, the biggest advantage of such systems of the physical locomotion and naturalness becomes a disadvantage after longer use.

The flying interaction technique with a joystick is often used as an alternative technique [Bowman2004]. It is the most common way to navigate in IVEs because of its simplicity and familiarity. Compared to physical locomotion techniques, joystick-based interaction techniques can be quickly designed and evaluated for desired virtual space. In addition, there is much less physical fatigue than using physical locomotion. However, according to Usoh et al. [Usoh1999], the joystick-based flying technique gives lower sense of presence, in terms of simplicity and naturalness, than physical locomotion technique, such as walking and walking in place.

Interaction techniques exploiting physical motion cues for navigation in VEs have been compared with the joystickbased technique to investigate two aspects: quality of presence [Slater1995, Usoh1999] and task performance related to spatial knowledge acquisition [Iwata1999, Peterson1998]. The former research takes the strategy that the more natural locomotion enhances the sense of presence, meaning that people may have the better spatial awareness. The latter shows that human can gain better spatial knowledge for navigation by integrating multisensory information. This information may come from proprioception, vestibular apparatus, and kinaesthetic sense, and vision.

The user interfaces of multi-touch devices, such as iPhone, iPod Touch, and Microsoft Surface, demonstrate innovative ways of objects manipulation. The Lemur device [JazzMutant.] was the first multi-touch commercial device which provided SDK. It is originally designed as an easily reconfigurable control surface for audio and media applications. As far as we know we were the first to use the Lemur device and iPhone/iPod touch for navigation in IVEs.

\section{NAVIGATION TECHNIQUES}

\section{Joystick-based flying}

'Flying', a common navigation technique in VEs, is based on the direction of a (hand manipulated) wand or based on the head orientation. In our experiment, we used the technique based on the wand orientation to determine the traveling direction. The joystick on the wand is used to translate and rotate the viewpoint. The buttons on the wand are used to control the flying speed.

\section{Two-handed FWIP}

The FWIP technique [Kim2008] was originally developed to use two hands to simultaneously operate translate and rotate the viewpoint. The basic idea is that our embodied resource, i.e., action-based navigation, can be mapped to 
finger-based interaction technique. In the case of the FWIP, our walking knowledge overlearned using two legs can be realized using our fingers to navigate in a virtual world and we can expect same (or at least similar) effects using fingers based on the 'walking' knowledge.

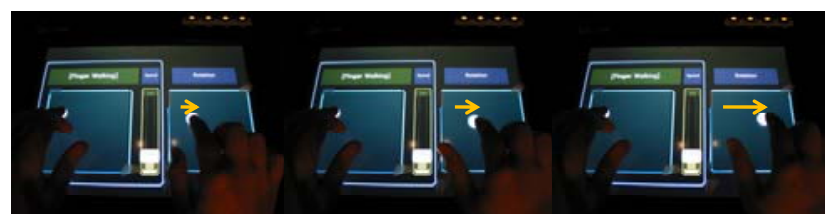

Figure 1. An example of the 'dragging' rotation-in-place technique. Dragging from the left to the right means rotating the world clockwise

In the previous study [Kim2008], we presented walking-inplace and rotation-in-place techniques to translate and rotate a viewpoint, respectively. Three different rotation-inplace techniques (i.e., 'walking', 'dragging' and 'jogdialing') were introduced and separately operated from the walking-in-place technique. Two rotation-in-place techniques (i.e., 'walking' and 'dragging') were evaluated (Figure 1). Since the interface is designed to separately operate both techniques (i.e., walking-in-place and rotation-in-place), the participants usually used two hands to rotate and transfer the viewpoint.

Since we observed that some of participants were confused with two separate operations, one of which is assigned to each hand, we developed a new one-handed FWIP technique, using the 'dragging' rotation-in-place technique, for this paper.

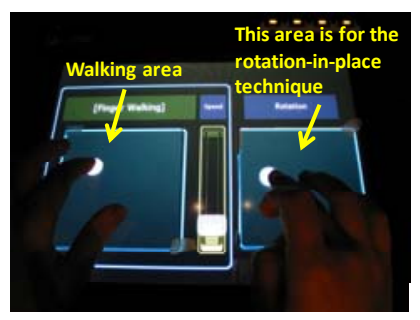

(a) Two-handed FWIP

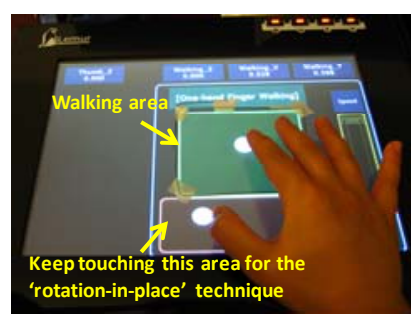

(b) One-handed FWIP
Figure 2. Interface design for the two-handed and the onehanded FWIP techniques.

\section{One-handed FWIP}

We re-designed the FWIP to allow users to use one hand to operate two techniques, walking-in-place and rotation-in place. Figure 2 shows different user interface designs for the two-handed (Figure 2(a)) and the one-handed FWIP techniques (Figure 2(b)). In order to combine with the walking-in-place technique, we chose the 'dragging' technique from the two rotation-in-place techniques evaluated in the previous study, because the previous study showed that the 'dragging' technique was manipulated with less confusing and less fatigue than the 'walking' technique [Kim2008].

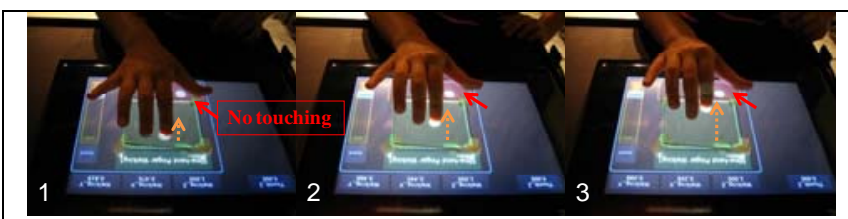

(a) Walking-in-place: walking forward

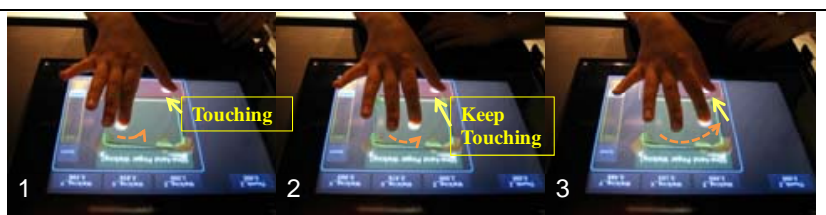

(b) Rotation-in-place: Rotate the world counter clockwise

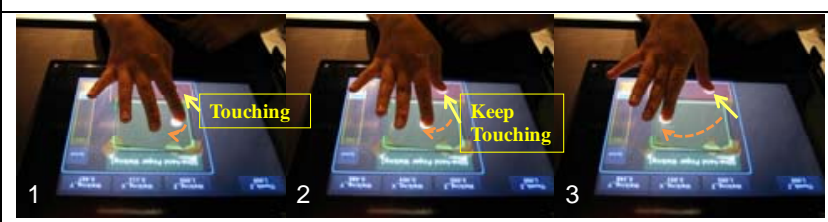

(c) Rotation-in-place: Rotate the world clockwise

Figure 3. One-handed FWIP technique.

As in the two-handed FWIP, the users can walk in any direction using their fingers (Figure 3(a)). For rotation-inplace, the users simply keep touching with the thumb on an area of the user interface separate from the walking area. The index finger (or another finger the user prefers) is used for dragging. Dragging to the left provides counterclockwise rotation (Figure 3(b)) while dragging to the right provides clockwise rotation (Figure 3(c)). The same technique with a little modification is also applied to iPhone/iPod Touch [Kim2009].

\section{COMPARATIVE STUDY}

The goal of the comparative study was to investigate the differences of the joystick's flying technique, the Lemurbased FWIP technique and the iPhone-based FWIP technique, for navigation tasks in VEs. The study was designed to compare "Control accuracy", "Route knowledge acquisition”, "Survey knowledge acquisition”, and "Task efficiency in spatial knowledge acquisition (using task completion time)" of these interaction techniques. We were especially interested in the spatial knowledge acquisition, rather than in control accuracy. We used the same experiment design presented in [Peterson1998] where Virtual Motion Controller (VMC) was compared with a joystick interface. There are two reasons we used their experiment design. First of all, the study showed that the experiment design is appropriate for users to travel both in terms of temporal and spatial sizes. The second reason is that the study showed the effects of the VMC using the Landmark-Route-Survey (LRS) model [Siegal1975]. Since our interest of spatial knowledge acquisition is based on the LRS model in order to investigate the wayfinding effect of our interaction techniques, we decided to use the experiment design of the VMC. 


\section{Experiment Design}

Joystick users would hold a wand device with a dominant hand and standing on a floor, point the wand where they would go, and control the joystick on the wand (Figure 4(a)). Lemur and iPhone devices are only used as a surface for finger-walking, and the navigation direction is only determined by finger-movement. In order to conduct the experiment with the constraint that the FWIP users would not physically move in the CAVE immersive space, we placed the Lemur and the iPhone/iPod Touch on a table to provide a persistent spatial reference. The FWIP users would stand on a floor next to the table. While the Lemur users would use only one-hand Figure 4(b)), iPhone/iPod Touch users would hold the device with the non-dominant hand, align it with the vertical line of the front wall in the CAVE space, and move their fingers with the dominanthand on the screen surface (Figure 4(c)).

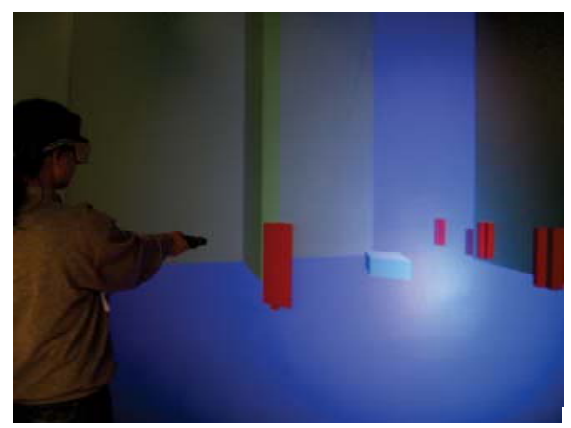

(a) Joystick user.

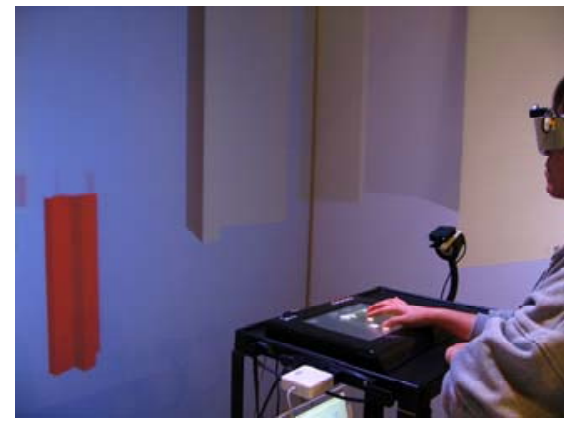

(b) Lemur user

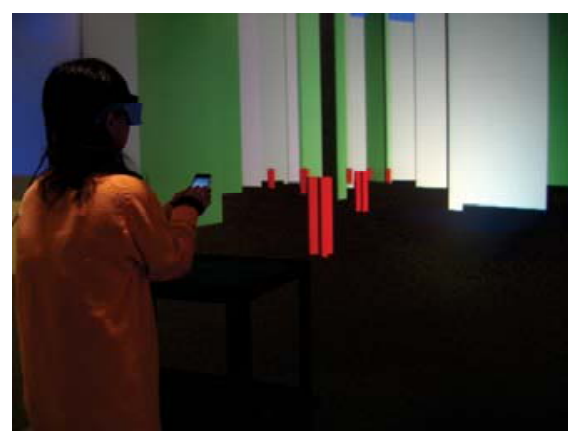

(c) iPhone user.

Figure 4. Experiment setup: the user is located in the CAVE area interacting with an immersive VE.

\section{Variables}

The experiment was designed using a three-by-two factorial. The independent variables were the interaction type and the maze type. Three different interaction techniques (i.e., joystick's flying, Lemur-based FWIP, and iPhone-based FWIP) were designed for the between-subjects study. Two different maze types (i.e., the simple and the complex mazes) were designed for a within-subjects study. The dependent variables were Control accuracy, Route knowledge acquisition error score, Survey knowledge acquisition error score, and Spatial-temporal error score (to examine "task efficiency in spatial knowledge”).

\section{Virtual World}

We created three mazes, including the practice maze, with the different complexities, and their details are described in Table 1.

\begin{tabular}{|l|c|c|c|}
\hline & $\begin{array}{l}\text { Practice } \\
\text { Maze }\end{array}$ & $\begin{array}{l}\text { Simple } \\
\text { Maze }\end{array}$ & $\begin{array}{l}\text { Complex } \\
\text { Maze }\end{array}$ \\
\hline \# of markers & 10 & 15 & 16 \\
\hline Size (model unit) & $265 \times 185$ & $245 \times 195$ & $230 \times 230$ \\
\hline $\begin{array}{l}\text { Route length } \\
\text { (model unit) }\end{array}$ & 213 & 310 & 377 \\
\hline $\begin{array}{l}\text { Cumulative angle } \\
\text { to turn (degrees) }\end{array}$ & 337 & 479 & 699 \\
\hline Fog effect & Yes & Yes & Yes \\
\hline
\end{tabular}

Table 1: The characteristics of the virtual mazes.

The practice maze (Figure 5(a)) is used to familiarize the subjects with the experiment procedure, and they would use to traverse the simple maze (Figure 5(b)) and the complex maze (Figure 5(c)).

The subjects can see the virtual objects (e.g., walls, marker or box objects) with different shadows due to the light effects while traveling in the mazes. Except for the light effect, we did not provide any visual feedback. For example, there is no color change nor object disappearance when a subject hits a marker object. This certainly imposes greater cognitive load on the subjects because they cannot rely on the visual feedback but need to depend on their own decisions.

However, it is intended for the subjects to move slowly, so that they can hit the marker objects precisely when they are close to the marker objects. Consequently, they have a chance to see the relations between the marker object and the other static objects, such as walls or box objects. Knowing these relations is important for the subject to recall the traveling route without the marker objects. 


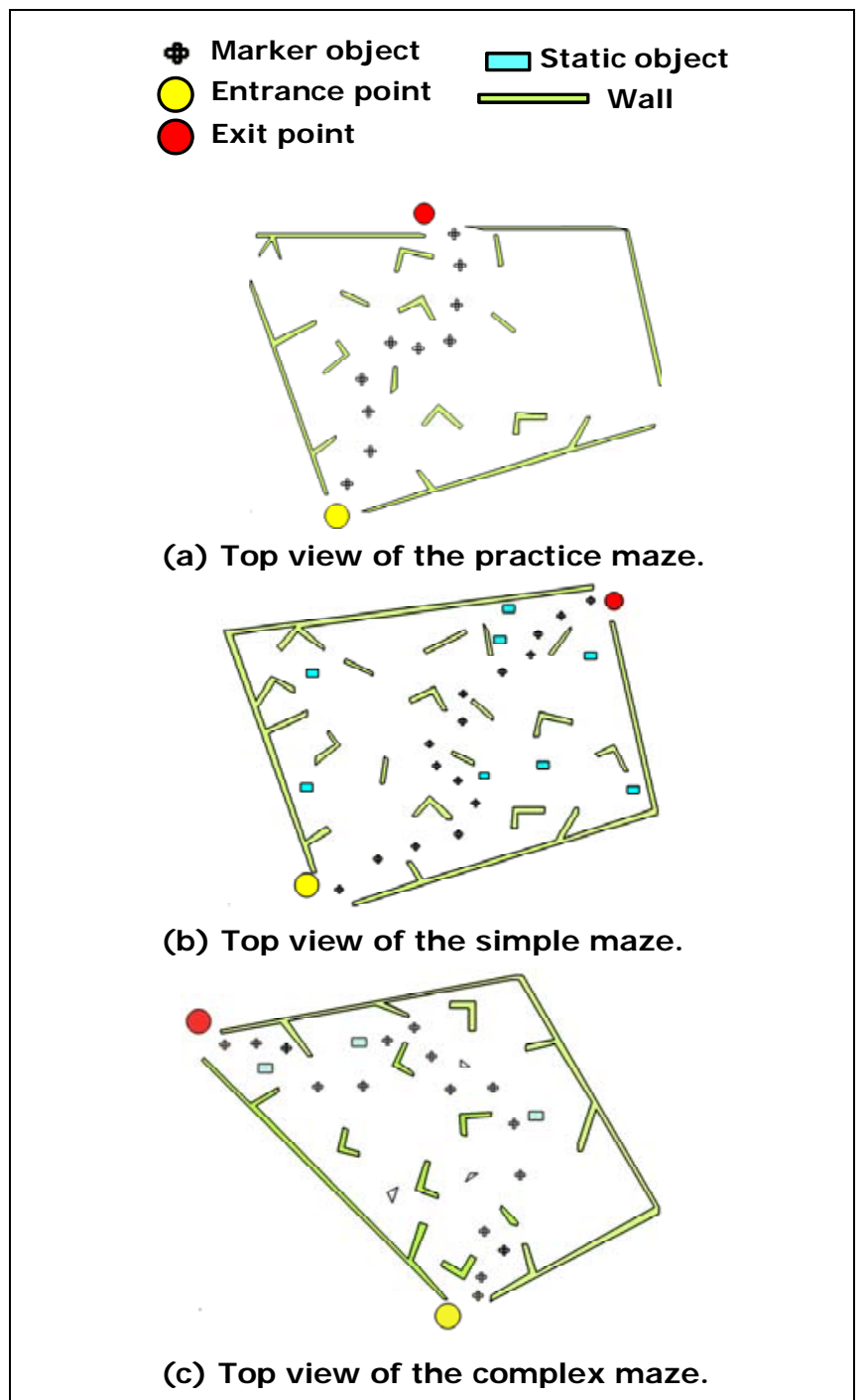

Figure 5. Virtual mazes: Each maze includes marker objects and walls. The simple and complex mazes also include some static objects that can be used for subjects remember their traveling paths.

\section{Procedure}

Forty eight college students participated in this experiment. The subjects were assigned to three different interaction groups: the joystick's flying group (JS group), the Lemurbased FWIP group (Lemur group), and iPhone-based FWIP group (iPhone group). The subjects were asked to fill out the pre-experiment questionnaire including demographic questions, such as age, gender, and VE experience level. The demographic data are summarized in Table 2.

\begin{tabular}{|c|c|c|c|}
\hline Data & J S group & Lemur group & iPhone Group \\
\hline $\begin{array}{l}\text { Mean } \\
\text { age } \\
\text { (years) }\end{array}$ & $\begin{array}{l}24.5 \\
(S t d=5.797)\end{array}$ & $\begin{array}{l}20.563 \\
(S t d=1.999)\end{array}$ & $\begin{array}{l}19.13 \\
(S t d=1.0888)\end{array}$ \\
\hline Gender & $\begin{array}{l}\text { Female: } 8, \\
\text { Male: } 8\end{array}$ & $\begin{array}{l}\text { Female: } 8, \\
\text { Male: } 8\end{array}$ & $\begin{array}{l}\text { Female: } 8, \\
\text { Male: } 8\end{array}$ \\
\hline $\begin{array}{l}\text { VE } \\
\text { experie } \\
\text { nce }\end{array}$ & $\begin{array}{l}\text { Non- } \\
\text { experienced } \\
\mathrm{N}=10, \\
\text { Experienced } \\
\mathrm{N}=6\end{array}$ & $\begin{array}{l}\text { Non- } \\
\text { experienced } \\
\mathrm{N}=10 \\
\text { Experienced } \\
\mathrm{N}=6\end{array}$ & $\begin{array}{l}\text { Non- } \\
\text { experienced } \\
\mathrm{N}=15, \\
\text { Experienced } \\
\mathrm{N}=1\end{array}$ \\
\hline
\end{tabular}

Table 2. Demographic data for the subjects.

All subjects of these groups were instructed on the experiment tasks, as follows:

1. Travel along the pre-defined route with marker objects five times (to have the experience of the maze environment): During these five trials, the subjects were asked to pass right through every marker object until they reach the exit. After each trial, the subjects were automatically moved back to the entrance point.

2. Estimation: After each trial, the subjects were asked how confidently they estimate the direction to the exit and how confidently they can replicate the same route without marker objects.

3. Route replication (for route knowledge acquisition): After five trials, the subjects had two trials to replicate the same route without visible marker objects.

4. Travel along the shortest path (for survey knowledge acquisition): After the route replication, the subjects had two trials to find the shortest path. When finding the shortest path, the subjects were allowed to walk through internal walls (no collision detection).

After all the tasks in three mazes, the post-experiment questionnaire obtained subjective responses to the experiment and free-form comments. The subjects were asked to describe the strategies employed to replicate the route and to find the shortest path to the exit. They were required to take a break after completing the tasks in each maze.

\section{ANALYSIS AND DISCUSSION}

\section{Measurements}

As aforementioned, we used the same experiment design and measurements of VMC, but our analysis should be different because the locomotion techniques of FWIP and the joystick are differently represented as 'walking' and 'flying', respectively, while the locomotion technique of VMC is represented same with the joystick as 'flying'.

Control accuracy. We investigate how precisely the subjects can control the interface for the traveling task with 
the number of hitting marker objects. Control accuracy is presented as an average hitting rate during the five trials.

Route knowledge acquisition error score. It is the area formed by the optimal path (the thick blue line in Figure 6(a)) and the user's traveling path during route replication tasks (Figure 6(a)). The route replication task was performed twice and the better result is used for data analysis because two results are dependent and each subject has a different learning curve. Consider that the data result showed that in the first trial ten subjects performed better in the simple maze and eight subjects performed better in the complex maze. These subjects may retain their route knowledge only for a short time. On the other hand, some subjects wandered much in the first trial, but they performed better in the second trial. We assumed that there are individual differences in gaining spatial knowledge, and so the better result from the two trials was chosen for data analysis. We shall denote this measure $\varepsilon_{R K}$.

Survey knowledge acquisition error score: It is the area formed by the optimal shortest path and the direct path taken by the user (Figure 6(b)). This task was also performed twice and the better result is used for data analysis. Consider that the data result showed that in the first trial five subjects performed better in the simple maze and seven subjects performed better in the complex mazes. In the case of the other subjects, they would realize that their estimation about the exit direction was wrong in the first trial, and could find the correct direction in the second trial. We shall denote this measure $\varepsilon_{S K}$

While learning curves vary from individual to individual, we did not go into details of the learning process. Instead we focused on the performance results. Our measurements included the subjective estimation of the confidence levels, regarding to point the exit direction and replicate the route path, and the error rate from users' drawing an arrow to express the users' estimation of the exit direction. However, we found no significance from the Pearsons correlation test in these measurements related to their objective performance. The regarded data analysis is not included in this paper.

Spatial-temporal error score: As an indicator to examine the task efficiency of each interaction technique in spatial knowledge acquisition, we used task completion time as well as spatial knowledge acquisition error scores. Since the error scores (i.e., $\varepsilon_{R K}$ and $\varepsilon_{S K}$ ) and the time factor (i.e., task completion time) are inversely propositional to the task efficiency, we calculate $\varepsilon_{R K} \times$ "task completion time" (for route knowledge acquisition) and $\varepsilon_{S K} \times$ "task completion time" (for survey knowledge acquisition), and denote these measures $\varepsilon_{R K^{*} \text { sec }}$ and $\varepsilon_{S K^{*} s e c}$, respectively.

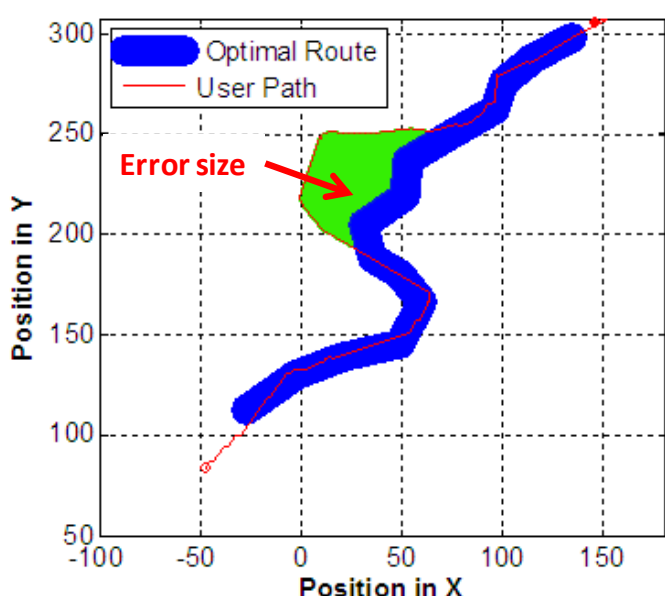

(a) The error size of route knowledge acquisition in the simple maze. The thickness of the optimal route is determined by the width of the marker objects.

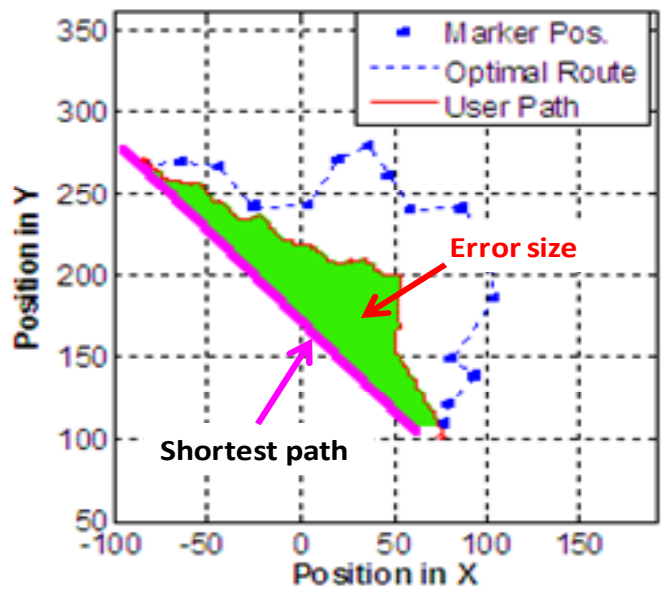

(b) The error size of survey knowledge acquisition in the complex maze.

Figure 6. Two examples of the error sizes of route and survey knowledge acquisition

\section{Results}

Control Accuracy

The result shows that users' control accuracy of hitting each marker object is better in the JS group. The average hitting rate is summarized in Table 3.

\begin{tabular}{|l|c|c|c|}
\hline & J S group & Lemur group & iPhone Group \\
\hline $\begin{array}{l}\text { Simple } \\
\text { Maze }\end{array}$ & $89.69(\%)$ & $80.75(\%)$ & $80.83(\%)$ \\
\hline $\begin{array}{l}\text { Complex } \\
\text { Maze }\end{array}$ & $90.15(\%)$ & $75.54(\%)$ & $79.84(\%)$ \\
\hline
\end{tabular}

Table 3. Hit rates of markers in simple and complex mazes

We conjecture that the result would be caused by the difference of controlling method to determine the moving 
direction. As shown in Figure 7, using the joystick, the user can continuously move (Figure 7(a)), meaning that the user can keep changing the direction. The path shows a continuous curve.

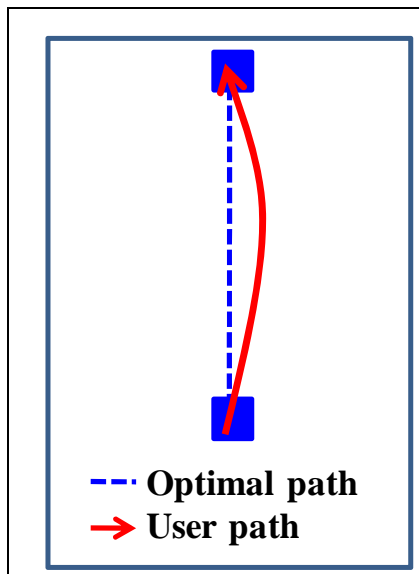

(a) Flying locomotion

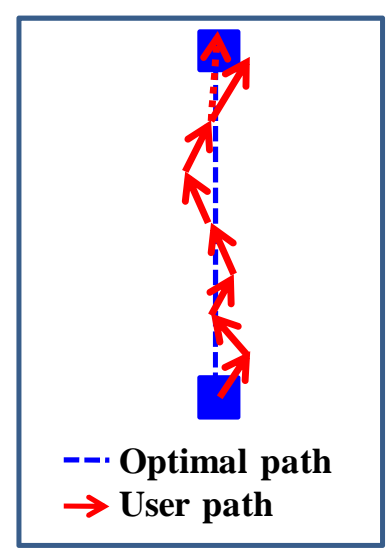

(b) Walking locomotion
Figure 7. Different movement of flying and walking locomotion

However, as shown in Figure 7(b), when using fingers (i.e., FWIP), the user moves step by step. It means that the user cannot change the direction until the step is completed. The discrete movement when using fingers may affect the result. The path shows a piecewise linear curve.

Due to the fact that human fingers are not mechanical controllers, the movement is not straight as much as the user would think. If the user did not carefully move fingers in the FWIP, the user is likely to skip the target marker object. Since the hit count is strictly recorded when the user actually hits the middle part of the marker object, it would not be recorded when the user slightly hits on the side of the object. The purpose of this strict record makes users try to remember the exact position of each marker object for the route replication task.

\section{Route Knowledge Acquisition}

For the comparison of users route knowledge acquisition, we calculated the area formed by two paths as an error score of how far the users moved from the optimal route (from the first marker to the last marker), and then normalized it using the largest error score, such that $\bar{\varepsilon}_{R K}=\varepsilon_{R K} / \max \left(\varepsilon_{R K}\right)$ in our data analysis. The results are shown in Figure 8.

Since there were two outliers (one user wandered too much and the other one was lost) in the JS group in the simple maze, we compared fourteen-user data for each interaction technique group.

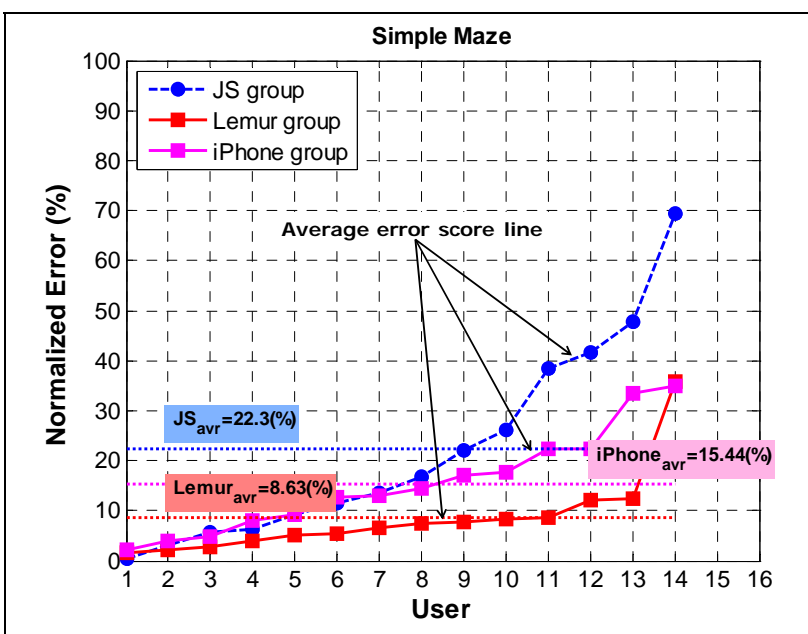

(a) Result in the simple maze

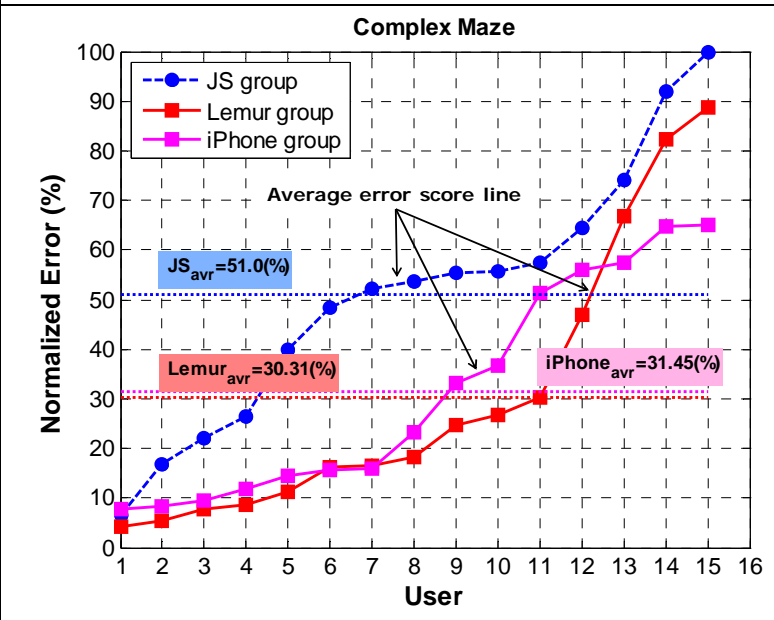

(b) Result in the complex maze

Figure 8. The comparison of $\bar{\varepsilon}_{R K}$ across our three groups. Even though the horizontal axis represents discrete users, we added the line between two discrete user data to easily compare the results of three groups (neither interpolation nor extrapolation).

In the simple maze (Figure 8(a)), the average error score of the JS group is a little bigger compared to the other groups. Because three-group samples failed the normality test (Ryan-Joiner $=0.789,0.801$, and 0.863, respectively, $p<$ $0.05)$, we used the Kruskal-Wallis non-parametric test $(\mathrm{H}$ statistic). This test shows significant difference among three groups' means $(\mathrm{H}=7.34, p<0.05)$. In the complex maze (Figure 8(b)), the average error score of the joystick user group is a little bigger compared to the other groups. Because the Lemur group samples failed the normality test (Ryan-Joiner=0.911, $p<0.05$ ), we used the Kruskal-Wallis non-parametric test ( $\mathrm{H}$ statistic). The result showed that there is no significant difference among three groups. However, when we compare the JS group vs. the Lemur group and the JS group vs. iPhone group using MannWhitney non-parametric test, the statistical tests show interesting results, as shown in Table 4. 


\begin{tabular}{|c|c|c|}
\hline & JS vs. Lemur & JS vs. iPhone \\
\hline Simple maze & $\mathrm{W}=281, \mathrm{p}<0.05$ & $\mathrm{~W}=245, \mathrm{p}>0.05$ \\
\hline Complex maze & $\mathrm{W}=284, \mathrm{p}<0.05$ & $\mathrm{~W}=277, \mathrm{p}<0.05$ \\
\hline
\end{tabular}

Table 4. Statistical test results for the comparison of route knowledge acquisition: JS group vs. Lemur group and JS group vs. iPhone group

Since the purpose of this study focuses on the comparison of the FWIP and the joystick's flying, we are more interested in the two-group based results as shown in Table 4.

Figure 8(a) shows that nine subjects in the JS group rank below the average error score line, while eleven subjects in the Lemur group rank below the line. In addition, twelfth and thirteenth subjects rank very close to the average error score line, which is not the case in the JS group. Thus, Figure 5(a) implies that the Lemur group performed evenly well against the JS group in the simple maze. On the other hand, the iPhone group is placed between the JS group and the Lemur group. Since the iPhone device should be held in non-dominant hand, its alignment may be sometimes off the vertical line of the front wall in the CAVE space. We conjecture that it may affect the task performance.

As shown in Table 4, the error score of the JS group is significantly greater than those of the other groups. Figure 8(b) shows that the performance of route knowledge acquisition was affected by the maze complexity. Thus, in the complex maze, both groups of the FWIP show the better performance on route knowledge acquisition.

\section{Survey Knowledge Acquisition}

In the same we did the route knowledge acquisition evaluation, we calculated the error score of how far the participants traveled from the optimal shortest path (from the entrance point to the exit point), and then normalized our $\varepsilon_{S K}$ using the largest error score, such that $\bar{\varepsilon}_{S K}=\varepsilon_{S K} / \max \left(\varepsilon_{S K}\right)$ in our data analysis. In both mazes, the average error scores of three groups are almost same, as shown in Table 5.

While the route knowledge is established from one marker to the next closest maker, the survey knowledge is formed to represent the overall map of the maze, from the entrance to the exit. Table 5 shows that the survey knowledge acquisition is not much affected by the interaction technique but the maze complexity.

\begin{tabular}{|c|c|c|c|}
\hline & JS group & $\begin{array}{c}\text { Lemur } \\
\text { group }\end{array}$ & $\begin{array}{c}\text { iPhone } \\
\text { group }\end{array}$ \\
\hline $\begin{array}{c}\text { Simple } \\
\text { maze }\end{array}$ & $20.81(\%)$ & $21.85(\%)$ & $20.16(\%)$ \\
\hline $\begin{array}{c}\text { Complex } \\
\text { maze }\end{array}$ & $54.27(\%)$ & $55.24(\%)$ & $57.0(\%)$ \\
\hline
\end{tabular}

Table 5. The average error score for survey knowledge.

\section{Task Efficiency}

Task completion Time: Each task does not have any time limit, so that the subjects can take their time to explore each maze. However, we observed that most subjects tried to quickly finish a task during the five trials because their next target is visible and they had no reason to explore the space to find the next target.

As shown in Table 6, the average traveling time of three techniques to complete a task during five trials is different. It means that each group users travel with different speed ${ }^{2}$.

\begin{tabular}{|l|c|c|c|}
\hline & JS group & $\begin{array}{c}\text { Lemur } \\
\text { group }\end{array}$ & $\begin{array}{c}\text { iPhone } \\
\text { group }\end{array}$ \\
\hline $\begin{array}{l}\text { Simple } \\
\text { Maze }\end{array}$ & 57.1 & 71.79 & 88.52 \\
\hline $\begin{array}{l}\text { Complex } \\
\text { Maze }\end{array}$ & 60.15 & 80.71 & 98.51 \\
\hline
\end{tabular}

Table 6. Average time (sec) of three techniques to complete a task

We illustrate the average time of three groups to complete a task in route replication and shortest path estimation tasks in both mazes in Table 7 .

\begin{tabular}{|c|c|c|c|c|}
\hline $\begin{array}{l}\text { Task } \\
\text { type }\end{array}$ & JS group & $\begin{array}{c}\text { Lemur } \\
\text { group }\end{array}$ & $\begin{array}{c}\text { iPhone } \\
\text { group }\end{array}$ & $\begin{array}{l}\text { Maze } \\
\text { type }\end{array}$ \\
\hline$R K$ & 57.18 & 46.4 & 56.61 & \multirow{2}{*}{$\begin{array}{c}\text { Simple } \\
\text { Maze }\end{array}$} \\
\hline SK & 16.76 & 17.59 & 24.14 & \\
\hline RK & 51.03 & 58.34 & 63.36 & \multirow{2}{*}{$\begin{array}{c}\text { Complex } \\
\text { Maze }\end{array}$} \\
\hline SK & 20.98 & 25.34 & 39.72 & \\
\hline
\end{tabular}

Table 7. Average time (sec) to complete a task in route replication and shortest path estimation tasks

Spatial-Temporal error score: Since the error scores (i.e., $\varepsilon_{R K}$ and $\varepsilon_{S K}$ ) and the time factor (i.e., task completion time) are inversely propositional to the task efficiency, we first calculate $\varepsilon_{R K^{*} s e c}=\varepsilon_{R K} \times$ "task completion time of route replication task" and $\varepsilon_{S K{ }^{*} s e c}=\varepsilon_{S K} \times$ "task completion time of shortest path estimation task" as the spatial-temporal error scores. Then we normalized each of our $\varepsilon_{R K^{*} \text { sec }}$ and $\varepsilon_{S K^{*} \mathrm{sec}}$ using the largest error score, such that $\bar{\varepsilon}_{R *^{*} s e c}=\varepsilon_{R *^{*} s e c} / \max \left(\varepsilon_{R K{ }^{*} s e c}\right)$ and $\bar{\varepsilon}_{S{ }^{*} s e c}=\varepsilon_{S K *^{*} s c} /$ $\max \left(\varepsilon_{S K^{*} s e c}\right)$ in our data analysis. Finally, we inverse the spatial-temporal error score to get the normalized efficiency.

Task efficiency in route knowledge acquisition: Figure 9 shows the efficiency result in route replication task.

\footnotetext{
2 The different speed of Lemur's FWIP and iPhone's FWIP is based on different implementation by considering different size of the device surface.
} 


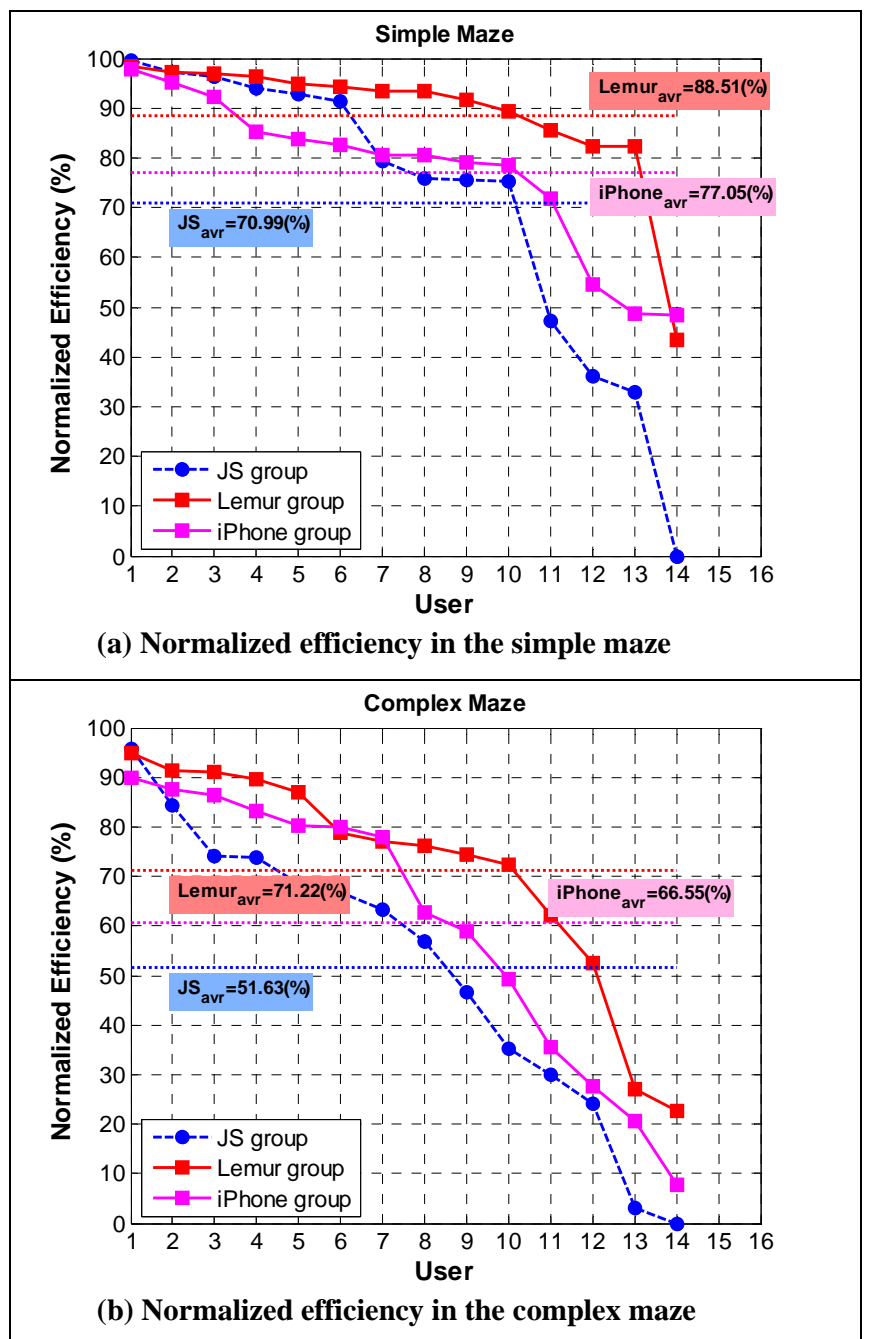

Figure 9. The comparison of the normalized efficiency across our three groups

Since there were two outliers in the joystick user group in both mazes, we compared fourteen-user data for each interaction technique group.

In the simple maze (Figure 9(a)), the average efficiency of the JS group is a little less compared to the other groups. Because the JS group and the Lemur group samples failed the normality test (Ryan-Joiner $=0.918$ and 0.779, respectively, $p<0.05$ ), we conducted Mann-Whitney nonparametric test whether or not there is significant difference between the JS group and the Lemur group and between the JS group and the iPhone group. The results show that there is no significant difference in two comparisons.

In the complex maze (Figure 9(b)), the JS group and the Lemur group samples failed the normality test (RyanJoiner=0.876 and 0.912, respectively, $p<0.05$ ). We conducted Mann-Whitney non-parametric test, and the results show that there is only significant difference between the JS group vs. the Lemur group in the complex maze $(\mathrm{W}=276.0, \mathrm{p}<0.05)$.
Task efficiency in survey knowledge acquisition: Figure 11 shows the efficiency result in shortest path estimation task.

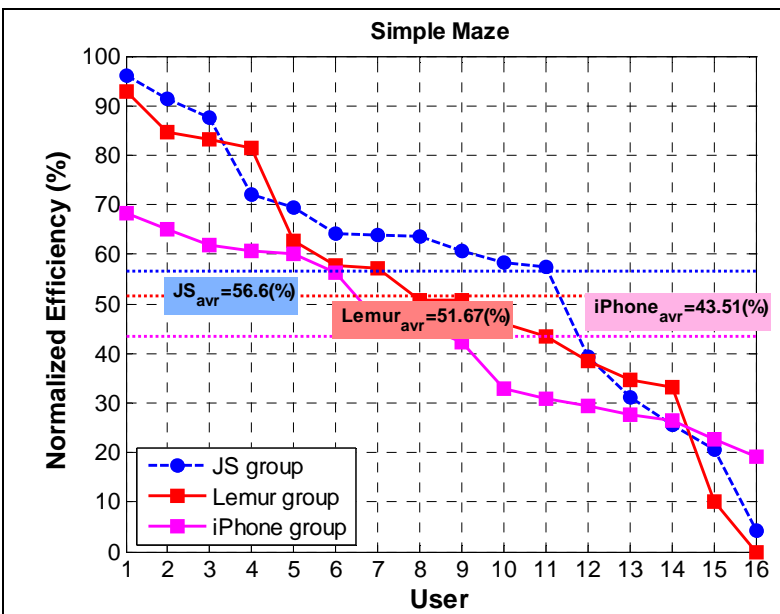

(a) Normalized efficiency in the simple maze

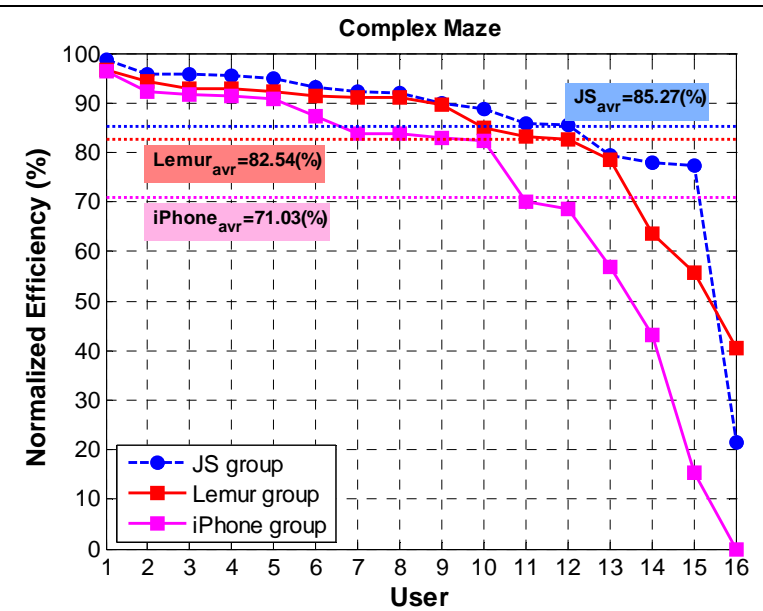

(b) Normalized efficiency in the complex maze

Figure 10. The comparison of the normalized efficiency across our three groups

In the simple maze (Figure 11(a)), three group samples follow normal distribution in the simple. We conducted ANOVA test. However, the results show that there is no significant difference between the JS group and the Lemur group and between the JS group and the Lemur group, respectively.

In the complex maze (Figure $1 \mathrm{a}(\mathrm{b})$ ), three group samples failed the normality test (Ryan-Joiner $=0.76,0.872$ and 0.883 , respectively, $p<0.05$ ). We conducted MannWhitney non-parametric test, and the results show that there is only significant difference between the JS group vs. the iPhone group in the complex maze $(\mathrm{W}=210.0, \mathrm{p}<0.05)$.

Discussion: We understand that it may be not meaningful to compare spatial-temporal error scores of three groups with different speed of three techniques. However, this task efficiency result indicates two things: 
P1: If a user explores a complex space where there are many turns, the FWIP may be the better technique in terms of spatial knowledge acquisition.

P2: If a user is already familiar with the space and the user travels a simple route (i.e., a straight path), the joystick's flying may be the better technique in terms of task efficiency.

In order to confirm $\mathbf{P 1}$ and $\mathbf{P} 2$, we need to perform more experiments thoroughly.

\section{Navigation strategies and user comments}

The navigation strategies to remember the path traveled and to find the exit point are different among individuals within each group.

\section{JS group}

We observed that the subjects used different behaviors for rotating their viewpoints during the first five trials with marker objects. These different behaviors are related to the next tasks of route replication and finding the shortest path without marker objects.

1. Without physically turning their bodies, some of the subjects only used the joystick to rotate the view point.

2. When holding the joystick close to their chests, some of the subjects physically turned their body in place to rotate the view point.

3. Some subjects physically turned their upper body while moving their arms together.

Even though the subjects showed different behaviors for spatial knowledge acquisition, the study results did not show which one is better for spatial knowledge acquisition. For example, there was an interesting behavior that some subjects tried a couple of different strategies. The subjects, who tried to physically turn their body to rotate their view point, were confused when trying to find the correct direction to go to the next marker object due to the indistinctive landmarks. These subjects changed their strategies to moving their arms, instead of turning their body.

Two subjects experienced in VR mentioned that the joystick is very friendly and intuitive to use because they are already familiar with it. However, it does not imply its usage is related to the navigation task.

On the other hand, two novice users complained about using the joystick because they felt that it took a while to get used to its flying movement and speed. However, their error scores of spatial knowledge acquisition are relatively low. That implies that the satisfaction of the interface may not be directly related to the performance of the spatial knowledge acquisition.

In terms of their strategies to remember the traveling route and find the exit direction, one half of the subjects in a group (i.e. eight subjects) mentioned that they tried to remember "where to turn" by using the relations between the other static objects and walls. In addition, some of them tried to remember the order of right turns or left turns. One user tried to use "elapsed-time estimation" of traveling between two markers. Another user tried to identify static objects and maze structures as a check point. To summarize, there are various strategies, but there is no strategy that works for everyone.

We also found that while most of the subjects had their own strategy for the route replication, they did not have any specific strategy for finding the shortest path. They would rather count on their estimation that might be improved during the repetitive trials including the route replication.

\section{FWIP group}

We first explained to them that the FWIP technique is designed using the analogy of the physical walking-in-place locomotion, as if they would walk on a treadmill. They were instructed about how to translate and rotate the view point with their finger movement on the device surface.

Based on the provided responses, the navigation strategies of this group can be described in two ways.

1. The subjects memorized the relations among objects (e.g., walls and box objects) and used them as reference points to go to the next spot.

2. The subjects counted and memorized the number of steps to walk and the number of turns to rotate from one spot to the next spot. Based on a subject, the spots can be where the marker objects were originally placed or they can be some static objects (e.g. walls and box objects).

Since the walking locomotion provides a constant movement speed, it was easier for the users to control their traveling speed compared to the joystick based flying interaction technique. While the speed with the joystick based flying technique is accelerated, it may take some time for users to get used to the speed, such as car-driving. The constant walking speed may help the subjects to unconsciously estimate the distance between marker objects after several repetitive trials. It can be effectively used when they replicated the traveling route without marker objects.

It is still premature to say whether or not these strategies are directly linked to acquiring better spatial knowledge because we do not know how well or bad would be their spatial knowledge acquisition without these strategies. However, we know that our proposed FWIP interaction technique allows them to develop these strategies. 


\section{SUMMARY AND FUTURE WORK}

This study is a first-step toward the investigation of the FWIP technique for spatial knowledge acquisition in an IVE. This study showed that a locomotion technique that provides a discrete and direct control over moving and turning (i.e., FWIP) results in better performance than rate based translation and turning (i.e., Joystick) in the route acquisition aspects of our maze navigation tasks. Since there some confounding factors, we cannot generalize our results. However, the results are useful, especially given how far the FWIP technique is removed from actual walking and turning. The results support the idea that the FWIP technique has merit, in terms of learning spatial knowledge, without any complex device, and is worth continued investigation. Using a relatively inexpensive consumer multi-touch device makes the FWIP technique more accessible and better suited for a widespread use.

We found that our experiment design had some open issues. For example, if the distribution of spatial ability in two groups had a significant difference, our conclusion could have been weaker. Another issue is that we only measured the partial aspect of survey knowledge because the subjects only explored one route. In future work, more carefully designed experiments will be used to address these issues and to remove confounders. In addition, we will explore the potential for using the FWIP technique in virtual reality (VR) applications, such as desktop VR and personalized VR interfaces (for example, using iPhones) in public spaces.

\section{REFERENCES}

1.Bourdot, $\mathrm{P}$ and Touraine, D. Polyvalent display framework to control virtual navigations by $6 \mathrm{DOF}$ tracking. In Proceedings of the IEEE Virtual Reality 2002 (VR’02), pages 277-278, 24-28 Mar. 2002.

2.Bowman, D.A., Kruijff, E. LaViola, Jr. J.J., and Poupyrev, I. 3D User Interfaces: Theory and Practice. AddisonWesley, Boston, 2004.

3.Camarata, K., Do, E. Y., Johnson, B. R., and Gross, M. D. Navigational blocks: navigating information space with tangible media. In Proceedings of the 7th international Conference on intelligent User interfaces. In Proc. IUI '02. ACM, New York, NY, 31-38. 2002.

4.Chance, S. S., Gaunet, F., Beall, A. C., and Loomis, J. M. Locomotion mode affects the updating of objects encountered during travel: The contribution of vestibular and proprioceptive inputs to path integration. Presence, 7, 168-178, 1998.

5.Cornell, E.H., Heth, C.D. and Skoczylas, M.J. The nature and use of route expectancies following incidental learning. Journal of Experimental Psychology, 19:209229, 1999.

6.Darken, R.P., Cockayne, W.R., and Carmein, D. The omni-directional treadmill: A locomotion device for virtual worlds. In Proc. of UIST1997, ACM Press (1997), 213-221.

7.Ittelson, W. H. Environment perception and contemporary perceptual theory. In W. H. Ittelson (Ed.), Environment and cognition (pp. 1-19). New York: Seminar Press, 1973.

8.H. Iwata and Y. Yoshida. Path reproduction tests using a torus treadmill. Presence: Teleoperators and Virtual Environments, 8(6):587- 597, Dec. 1999.

9.JazzMutant. Lemur, http://www.jazzmutant.com/, last accessed 30 Dec. 2008.

10.Kim, J., Gracanin, D., Matkovic, K., and Quek, F. Finger Walking in Place (FWIP): A Traveling Technique in Virtual Environments. In Proc. of $9^{\text {th }}$ International Symposium, Smart Graphics 2008, 58-69, 2008.

11.Kim, J., Gracanin, D., Matkovic, K., and Quek, F. iPhone/iPod Touch as Input Devices for Navigation in Immersive Virtual Environments, To appear in Proc. of Virtual Reality 2009.

12.LaViola, J. J., Feliz, D.A., Keefe, D.F. and Zeleznik, R.C. Hands-free multi-scale navigation in virtual environments. In Proceedings of the 2001 symposium on Interactive 3D graphics, pages 9-15. ACM Press, 2001.

13.Peterson, B., Wells, M., Furness III, T.A., and Hunt, E. The effects of the interface on navigation in virtual environments. In Proc. of the Human Factors and Ergonomics Society Annual Meeting, 1496-1505, 1998.

14.Siegel, A. W. and White, S. H. The development of spatial representations of large-scale environments. In H.W. Reese, editor, Advances in Child Development and Behavior, volume 10, 9-55. Academic, New York, 1975.

15.Slater, M., Usoh, M., and Steed, A. Taking steps: the influence of a walking technique on presence in virtual reality. ACM Transactions on Computer-Human Interaction 2, 3 (1995), 201-219.

16.Stankiewicz, B.J. and Eastman, K. (Accepted Pending Revision, 2008). Lost in Virtual Space II: The Role of Proprioception and Discrete Actions when Navigating with Uncertainty. Association for Computing Machinery/ Transactions on Applied Perception, 2008.

17.Templeman, J., Denbrook, P.S., and Sibert, L.E. Virtual locomotion: walking-in-place through virtual environments. Presence: Teleoperators and Virtual Environments 8, 6 (1999), 598-617.

18.Tlauka, M., Donaldson, P., and Wilson, D. Forgetting in spatial memories acquired in a virtual environment. Applied Cognitive Psychology, 22, 69-84, 2008.

19.Virginia Tech, VT-CAVE, http://www.cave.vt.edu/, last accessed 30 Dec. 2008.

20.Virtusphere, Inc. VirtuSphere. http://www.virtusphere.com/. Last accessed 31 Oct. 2008. 